\title{
Macrofungi determined in Yuvacık Basin (Kocaeli) and its close environs
}

\author{
Ilgaz AKATA ${ }^{1 *}$, Şanlı KABAKTEPE ${ }^{2}$, Mustafa SEVINDİK ${ }^{3}$, Hasan AKGÜL ${ }^{3}$ \\ ${ }^{1}$ Ankara University, Faculty of Science, Department of Biology, Tandoğan, Ankara, TURKEY \\ ${ }^{2}$ Inonu University, Battalgazi Vocational School, Battalgazi, Malatya, TURKEY \\ ${ }^{3}$ Akdeniz University, Faculty of Sciences, Department of Biology, Antalya, TURKEY \\ *Corresponding author: fungus@ hotmail.com.tr
}

Received Date: 17.01.2018

Accepted Date: 30.05 .2018

\section{Abstract}

Aim of the study: The purpose of this study was to determine the macrofungal diversity of Yuvacik Basin (Kocaeli) and its close environs.

Study area: The study area is situated in the east part of Marmara region within the boundaries of Kocaeli and Sakarya provinces of Turkey and it covers 25.759 hectares. The altitude range of region is 150-1610 meters. In addition, the region is in the transition zones of the three different phytogeographic regions containing the Euro-Siberian, the Mediterranean and Irano-Turanian

Material and Method: The fungal samples were collected from different localities of Yuvacik Basin and its close environs between 2015 and 2017. During field studies, macroscopic and ecological features of the samples were recorded and they were photographed in their natural habitats and then brought to the labratory. In the labaratory, macroscopic and microscopic characteristics of the samples were examined. After obtaining relevant macroscopic and microscopic data, fungal samples were identified. The identified samples were kept at the herbarium of Ankara University (ANK).

Main Results: As a result of field and laboratory studies, a total of 140 species belonging to 44 family and 14 order within the 2 divisions were listed. Among them, 13 species belonged to Ascomycota, 127 to Basidiomycota.

Research highlights: In this study, macrofungal diversity of Yuvacık Basin and its close environs were determined. One hundred and thirty-nine species were new for the region. Moreover, edible and poisonous macrofungi species were emphasized.

Keywords: Macrofungi, Biodiversity, Yuvacık Basin, Turkey

\section{Yuvacık Havzası (Kocaeli) ve yakın çevresinden belirlenen}

\section{makrofunguslar}

Özet

Çalışmanın amacı: Bu çalışmanın amacı, Yuvacık Havzası (Kocaeli) ve yakın çevrelerindeki makrofungal çeşitliliğini ortaya çıkarmaktır.

Çalışma alanı: Araştırma alanı, Marmara bölgesinin doğu kesiminde, Kocaeli ve Sakarya illeri sınırları içerisinde olup 25.759 hektarlık bir alanı kapsamaktadır. Alanın yükseklik aralığı 150-1610 metre arasında değişir. Buna ek olarak, çalışma alanı Avrupa-Sibirya, Akdeniz ve İran-Turan olmak üzere üç farklı fitocoğrafik bölgenin geçiş zonunda yer almaktadır

Materyal ve Yöntem: Mantar örnekleri 2015-2017 yılları arasında Yuvacık Havzası ve yakın çevrelerinden toplanmıştır. Arazi çalışmaları sırasında örneklerin makroskopik ve ekolojik özellikleri kaydedilmiş ve doğal ortamlarında fotoğraflanmıştır ve daha sonra bu örnekler laboratuvara getirilmiştir. Laboratuvarda örneklerin makroskopik ve mikroskopik özellikleri incelenmiştir. Gerekli makroskopik ve mikroskobik veriler elde edildikten sonra, mantar örnekleri teşhis edilmiştir. Teşhis edilen örnekler, Ankara Üniversitesi (ANK) herbaryumunda saklanmaktadır.

Temel Sonuçlar: Arazi ve laboratuvar çalışmalarının sonucunda, 2 bölüm, 14 ordo ve 44 familya içinde dağılım gösteren 140 tür listelenmiştir. Bunların arasında 13 tür Ascomycota, 127 tür ise Basidiomycota' ya aittir.

Araştırma vurguları: Bu çalışmada, Yuvacık Havzası ve yakın çevresinin makrofungal çeşitliliği ortaya çıkarılmıştır. Yüz otuz dokuz tür bölge için yenidir. Ayrıca, yenilebilir ve zehirli makrofungus türlerine vurgu yapılmıştır.

Anahtar Kelimeler: Makrofunguslar, Biyoçeşitlilik, Yuvacık Havzası, Türkiye 


\section{Introduction}

Yuvacik Basin is situated in the east part of Marmara region within the boundaries of Kocaeli and Sakarya provinces of Turkey (Figure 1). The basin, which is located in the A2 and A3 grid (see Davis 1965), covers 25.759 hectares with elevation varying between 150 and 1610 meters. It is in the transition zones of the three different phytogeographic regions including the EuroSiberian, the Mediterranean and Irano-
Turanian (Efe et al., 2013; Keten et al., 2010).

The region is characterized by transitional climate between the climates of the Mediterranean and the Black Sea (Çobanoğlu et al., 2013). The average annual precipitation is $1038.7 \mathrm{~mm}$ with December $(159,5 \mathrm{~mm})$ being the heaviest month. The average annual temperature is $9.5^{\circ} \mathrm{C}$ and the coldest and the warmest months of year are january and july respectively (Efe et al., 2013).

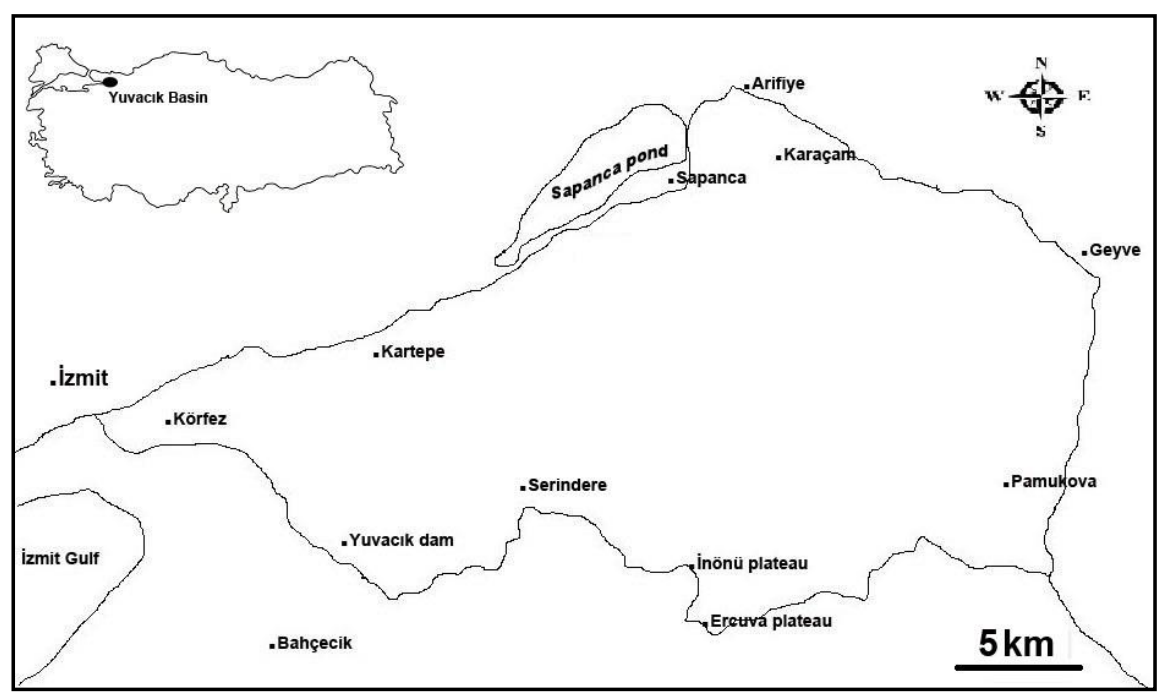

Figure 1. Map of the research area

The research area consists of agricultural areas, pastures, scrub and forests. The forest vegetation is dominated by fir, sometimes forming mixed stands with beech, Scots pine, black pine and oak. Some other trees such as common hornbeam, chestnut, common hazel, alder, poplar, walnut are also seen in the research area (Beşkardeş, 2012).

Numerous studies have been carried out on Turkish macromycota and some of them are still in progress (Akata, 2017; Akata and Uzun, 2017; Akata et al., 2015a,b, Alkan et al, 2016; Allı et al., 2017; Altuntaş et al., 2017; Demirel et al., 2016; Doğan and Kurt, 2016; Öztürk et al, 2017; Sesli and Denchev, 2008; Sesli et al., 2016). According to the present literature, some investigations on macrofungal taxonomy around the research area were carried out by Akata (2017), Allı et al. (2017), Karamanoğlu and Öder (1973), Öztürk et al. (1990), Solak and Gücin, 1992; Stojchev et al. (1998). But there is not any detailed study on macrofungal diversity of Yuvacık Basin and its surrounding area.

\section{Materials and Methods}

Macrofungi samples were collected from Yuvacık Basin (Kocaeli and Sakarya) between 2015 and 2017. During the field studies, macroscopic and ecological features of the samples were noted and they were photographed in their natural habitats. Macroscopic and microscopic characteristics of the samples, as well as their characteristic reactions against some chemical reagents were examined. The reagents such as distilled water, Melzer's reagent, $5 \% \mathrm{KOH}$, $\mathrm{H}_{2} \mathrm{SO}_{4}$, Cotton blue, Congo red etc. were used. Identification was performed with the aid of the literature (Breitenbach and Kränzlin, 1986-2000; Hansen and Knudsen, 1992-2000; Ryvarden and Gilbertson, 1993; Pegler et al., 1997; Calonge, 1998; Heilmann-Clausen et al., 1998; Kränzlin, 
2005). All samples were deposited to Ankara University Herbarium (ANK).

\section{Results}

The systematic of the taxa was in accordance with Cannon and Kirk (2008), Kirk et al. (2008), and Index fungorum (www.indexfungorum.org: accessed 1 November 2016). The taxa were listed in alphabetical order and they were presented together with notes on habitat, locality, collection date, geographical position, and accesion numbers (I. Akata: A.; Ş. Kabaktepe: K.; H. Akgül: HA.; M. Sevindik: S.).

\section{Systematic overview \\ Fungi \\ Ascomycota \\ Helotiales \\ Helotiaceae}

1. Ascocoryne cylichnium (Tul.) Korf: İnönü plateau, on beech stump, $1100 \mathrm{~m}, \mathrm{~N}$ $40^{\circ} 33^{\prime}$ - E $30^{\circ} 00^{\prime}, 25.10 .2015$, A\&HA. 080.

2. Bisporella citrina (Batsch) Korf \& S.E. Carp.: Serindere, on fallen beech branch, $900 \mathrm{~m}, \mathrm{~N} 40^{\circ} 37^{\prime}$ - E $30^{\circ} 00^{\prime}$, 27.10.2015, A\&HA. 115; İnönü plateau, on fallen beach branch, $1130 \mathrm{~m}, \mathrm{~N} 40^{\circ} 34^{\prime}-\mathrm{E}$ $29^{\circ}$ 59', 15.09.2016, A. 6471; İnönü plateau, on beech stump, $1120 \mathrm{~m}, \mathrm{~N} 40^{\circ} 33^{\prime}-\mathrm{E} 30^{\circ}$ 01', 01.11.2017, A\&K\&S. 115.

3. Hymenoscyphus serotinus (Pers.) W. Phillips: İnönü plateau, on fallen beech branch, $1170 \mathrm{~m}, \mathrm{~N} 40^{\circ} 34^{\prime}-\mathrm{E} 30^{\circ} 00^{\prime}$, 30.10.2015, A\&HA. 225.

\section{Leotiaceae}

4. Leotia lubrica (Scop.) Pers.: İnönü plateau, in fir and beech mixed forest, 1120 $\mathrm{m}, \mathrm{N} 40^{\circ} 33^{\prime}-\mathrm{E} 30^{\circ} 00^{\prime}, 29.10 .2015$, A\&HA. 210.

\section{Pezizales}

\section{Helvellaceae}

5. Helvella crispa (Scop.) Fr.: Yuvacik Dam, near road, $190 \mathrm{~m}, \mathrm{~N} 40^{\circ} 38^{\prime}$ - E $29^{\circ} 56^{\prime}$, 02.10.2015, A\&HA. 057.

6. Helvella elastica Bull.: İnönü plateau, in fir and beech mixed forest, 1170 $\mathrm{m}, \mathrm{N} 40^{\circ} 34^{\prime}$ - E $30^{\circ} 00^{\prime}, 30.10 .2015$, A\&HA. 212.

\section{Pezizaceae}

7. Peziza domiciliana Cooke: Karaaslan recreational area, near stream, $290 \mathrm{~m}, \mathrm{~N} 40^{\circ}$ $38^{\prime}$ - E $29^{\circ} 56^{\prime}, 28.10 .2015$, A\&HA. 151.

\section{Pyronemataceae}

8. Aleuria aurantia (Pers.) Fuckel: Ercova plateau, in fir forest, $1160 \mathrm{~m}, \mathrm{~N} 40^{\circ}$ 32' - E 29 56', 01.10.2015, A\&HA. 024.

9. Otidea onotica (Pers.) Fuckel: Pamukova, in fir forest, $600 \mathrm{~m}, \mathrm{~N} 40^{\circ} 32^{\prime}$ - E $30^{\circ} 06^{\prime}, 30.10 .2015$, A\&HA. 265.

\section{Xylariales}

\section{Xylariaceae}

10. Hypoxylon fragiforme (Pers.) J. Kickx f.: İnönü plateau, on beech stump, $1260 \mathrm{~m}, \mathrm{~N} 40^{\circ} 34^{\prime}$ - E $29^{\circ}$ 59', 15.09.2016, A. 6469.

11. Kretzschmaria deusta (Hoffm.) P.M.D. Martin: İnönü plateau, on beech stump, $1140 \mathrm{~m}, \mathrm{~N} 40^{\circ} 34^{\prime}$ - E $30^{\circ} 00^{\prime}$, 10.10.2016, A. 6539.

12. Xylaria hypoxylon (L.) Grev.: İnönü plateau, on beech stump, $1170 \mathrm{~m}, \mathrm{~N} 40^{\circ} 33^{\prime}$ E $30^{\circ} 00^{\prime}, 29.10 .2015$, A\&HA. 168; Kartepe, on beech stump, $254 \mathrm{~m}, \mathrm{~N} 40^{\circ} 41^{\prime}-\mathrm{E} 30^{\circ}$ 00', 30.10.2017, A\&K\&S. 070; Serindere, on apple stump, $480 \mathrm{~m}, \mathrm{~N} 40^{\circ} 38^{\prime}$ - E $30^{\circ} 01^{\prime}$, 01.11.2017, A\&K\&S. 123.

13. Xylaria polymorpha (Pers.) Grev.: İnönü plateau, on beech stump, $1135 \mathrm{~m}, \mathrm{~N}$ $40^{\circ} 33^{\prime}$ - E $30^{\circ} 00^{\prime}, 14.09 .2016$, A. 6457.

\section{Basidiomycota}

Agaricales

Agaricaceae

14. Coprinus comatus (O.F. Müll.) Pers.: Yuvacık Dam, near road, $180 \mathrm{~m}, \mathrm{~N} 40^{\circ} 38^{\prime}$ E 295', 02.10.2015, A\&HA. 051; Ercova plateau, near fir forest, $1170 \mathrm{~m}, \mathrm{~N} 40^{\circ} 31^{\prime}$ - E $29^{\circ} \quad 56, \quad 29.10 .2017, \quad$ A\&K\&S. 002; Serindere, near road, $489 \mathrm{~m}, \mathrm{~N} 40^{\circ} 38^{\prime}-\mathrm{E}$ $29^{\circ}$ 59', 30.10.2017, A\&K\&S. 043; Kartepe,


30.10.2017, A\&K\&S. 056.

15. Crucibulum laeve (Huds.) Kambly: Ercova plateau, on fir stump, $1180 \mathrm{~m}, \mathrm{~N} 40^{\circ}$ $32^{\prime}$ - E $29^{\circ} 56^{\prime}, 01.10 .2015$, A\&HA. 023.

16. Cyathus striatus (Huds.) Willd.: İnönü plateau, on fallen beech branch, 1120 $\mathrm{m}, \mathrm{N} 40^{\circ} 33^{\prime}$ - E 30 00', 29.10.2015, A\&HA. 194.

17. Cystoderma amianthinum (Scop.) Fayod: İnönü plateau, near fir forest, $1140 \mathrm{~m}$, N 40 $33^{\prime}$ - E $29^{\circ} 59^{\prime}, 26.10 .2015$, A\&HA. 103.

18. Cystoderma carcharias (Pers.) Fayod: Ercova plateau, in fir forest, $940 \mathrm{~m}, \mathrm{~N}$ $40^{\circ} 34^{\prime}$ - E $29^{\circ} 56^{\prime}, 01.10 .2015$, A\&HA 034. 
19. Lepiota castanea Quél.: İnönü plateau, in fir and beech mixed forest, 1100 $\mathrm{m}, \mathrm{N} 40^{\circ} 33^{\prime}$ - E $30^{\circ} 00^{\prime}, 25.10 .2015$, A\&HA. 076.

20. Lepiota cristata (Bolton) P. Kumm.: İnönü plateau, in meadow, $1180 \mathrm{~m}, \mathrm{~N} 40^{\circ} 34^{\prime}$ - E $30^{\circ} 00^{\prime}, 30.09 .2015$, A\&HA. 002.

21. Lepiota clypeolaria (Bull.) P. Kumm.: İnönü plateau, in fir and beech mixed forest, $1125 \mathrm{~m}, \mathrm{~N} 40^{\circ} 33^{\prime}$ - E $30^{\circ} 00^{\prime}$, 29.10.2015, A\&HA. 198.

22. Lepiota ignivolvata Bousset \& Joss. Ex Joss.: İnönü plateau, in fir and beech mixed forest, $1160 \mathrm{~m}, \mathrm{~N} 40^{\circ} 34^{\prime}-\mathrm{E} 30^{\circ} 00^{\prime}$, 30.10.2015, A\&HA. 217.

23. Leucoagaricus leucothites (Vittad.) Wasser: Balaban, in meadow, $200 \mathrm{~m}, \mathrm{~N} 40^{\circ}$ $41^{\prime}$ - E $30^{\circ} 05^{\prime}, 02.10 .2015$, A\&HA. 070.

24. Lycoperdon echinatum Pers.: İnönü plateau, in fir and beech mixed forest, 1120 $\mathrm{m}, \mathrm{N} 40^{\circ} 33^{\prime}-\mathrm{E} 30^{\circ} 00^{\prime}, 25.10 .2015$, A\&HA. 081.

25. Lycoperdon excipuliforme (Scop.) Pers.: İnönü plateau, fir and beech mixed forest edge, $1175 \mathrm{~m}, \mathrm{~N} 40^{\circ} 34^{\prime}$ - E $30^{\circ} 00^{\prime}$, 30.10.2015, A\&HA. 220.

26. Lycoperdon molle Pers.: İnönü plateau, fir forest edge, $1150 \mathrm{~m}, \mathrm{~N} 40^{\circ} 34^{\prime}$ - E $29^{\circ} 59^{\prime}, 28.10 .2015$, A\&HA. 146.

27. Lycoperdon perlatum Pers.: İnönü plateau, fir forest edge, $1170 \mathrm{~m}, \mathrm{~N} 40^{\circ} 34^{\prime}$ - E $30^{\circ} 00^{\prime}, 30.09 .2015$, A\&HA. 007.

28. Lycoperdon pyriforme Schaeff.: Ercova plateau, on fir stump, $900 \mathrm{~m}, \mathrm{~N} 40^{\circ}$ 34 ' - E $29^{\circ} 56^{\prime}, 01.10 .2015$, A\&HA. 037.

29. Lycoperdon utriforme Bull.: İnönü plateau, in meadow, $1170 \mathrm{~m}, \mathrm{~N} 40^{\circ} 34^{\prime}-\mathrm{E}$ $30^{\circ} 00^{\prime}, 30.09 .2015$, A\&HA. 001.

30. Macrolepiota konradii (Huijsmanex P.D. Orton) M.M. Moser: İnönü plateau, in fir and beech mixed forest edge, $1250 \mathrm{~m}, \mathrm{~N}$ $40^{\circ} 34^{\prime}$ - E $29^{\circ} 59^{\prime}, 10.10 .2016$, A. 6526.

31. Macrolepiota mastoidea (Fr.) Singer: İnönü plateau, in fir and beech mixed forest, $1125 \mathrm{~m}, \mathrm{~N} 40^{\circ} 33^{\prime}$ - E $30^{\circ} 00^{\prime}, 29.10 .2015$, A\&HA. 182.

32. Macrolepiota procera (Scop.) Singer: İnönü plateau, in fir and beech mixed forest edge, $1170 \mathrm{~m}, \mathrm{~N} 40^{\circ} 34^{\prime}$ - E $30^{\circ} 00^{\prime}$, 30.10.2015, A\&HA. 211; İnönü plateau, in fir forest, $1085 \mathrm{~m}, \mathrm{~N} 40^{\circ} 34^{\prime}-\mathrm{E} 30^{\circ} 01^{\prime}$, 30.10.2017, A\&K\&S. 060; Ercova plateau, in fir forest, $1140 \mathrm{~m}, \mathrm{~N} 40^{\circ} 34^{\prime}-\mathrm{E} 30^{\circ} 01^{\prime}$, 30.10.2017, A\&K\&S. 080.

\section{Amanitaceae}

33. Amanita citrina Pers.: İnönü plateau, in fir forest, $1140 \mathrm{~m}, \mathrm{~N} 40^{\circ} 33^{\prime}-\mathrm{E} 29^{\circ} 59^{\prime}$, 26.10.2015, A\&HA. 108.

34. Amanita muscaria (L.) Lam.: Ercova plateau, in fir forest, $1150 \mathrm{~m}, \mathrm{~N} 40^{\circ} 32^{\prime}-\mathrm{E}$ $29^{\circ} 55^{\prime}, 01.10 .2015$, A\&HA. 008; Ercova plateau, in fir forest, $1140 \mathrm{~m}, \mathrm{~N} 40^{\circ} 34^{\prime}-\mathrm{E}$ $30^{\circ} 01 ', 30.10 .2017$, A\&K\&S. 081; İnönü plateau, in fir and beech forest, $1110 \mathrm{~m}, \mathrm{~N}$ $40^{\circ} 33^{\prime}$ - E $30^{\circ} 01^{\prime}, 01.11 .2017$, A\&K\&S. 099 .

35. Amanita pantherina (DC.) Krombh.: Ercova plateau, in fir forest, $920 \mathrm{~m}, \mathrm{~N} 40^{\circ}$ 32' - E $29^{\circ} 56^{\prime}, 01.10 .2015$, A\&HA. 033.

36. Amanita phalloides (Vaill. ex Fr.) Link: İnönü plateau, in fir and beech mixed forest, $1110 \mathrm{~m}, \mathrm{~N} 40^{\circ} 33^{\prime}$ - E $30^{\circ} 00^{\prime}$, A\&HA. 074.

37. Amanita rubescens Pers.: Ercova plateau, in fir forest, $910 \mathrm{~m}, \mathrm{~N} 40^{\circ} 34^{\prime}-\mathrm{E}$ $29^{\circ} 56^{\prime}, 01.10 .2015$, A\&HA. 035.

38. Amanita vaginata (Bull.) Lam.: İnönü plateau, in fir and beech mixed forest edge, $1250 \mathrm{~m}, \mathrm{~N} 40^{\circ} 34^{\prime}-\mathrm{E} 29^{\circ} 5^{\prime}$, 10.10.2016, A. 6530.

\section{Cortinariaceae}

39. Cortinarius caperatus (Pers.) Fr.: Pamukova, in fir forest, under bilberry, 900

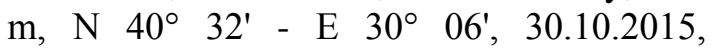
A\&HA. 263.

\section{Fistulinaceae}

40. Fistulina hepatica (Schaeff.) With.: İnönü plateau, on beech stump, $1135 \mathrm{~m}, \mathrm{~N}$ $40^{\circ} 33^{\prime}$ - E $30^{\circ} 00^{\prime}, 10.10 .2016$, A. 6516.

\section{Hydnangiaceae}

41. Laccaria amethystina Cooke: Ercova plateau, in fir forest, $1160 \mathrm{~m}, \mathrm{~N} 40^{\circ} 32^{\prime}-\mathrm{E}$ $29^{\circ} 55^{\prime}, 01.10 .2015$, A\&HA. 009.

42. Laccaria laccata (Scop.) Cooke: İnönü plateau, in fir forest, $1130 \mathrm{~m}, \mathrm{~N} 40^{\circ}$ 33' - E 29० 59', 26.10.2015, A\&HA. 107; Ercova plateau, in fir forest, $1085 \mathrm{~m}, \mathrm{~N} 40^{\circ}$ 34' - E $30^{\circ} 01 ', 30.10 .2017$, A\&K\&S. 077; Ercova plateau, in fir forest, $1102 \mathrm{~m}, \mathrm{~N} 40^{\circ}$ $33^{\prime}$ - E $30^{\circ} 01^{\prime}, 30.10 .2017$, A\&K\&S. 094; İnönü plateau, in fir forest, $1130 \mathrm{~m}, \mathrm{~N} 40^{\circ} 32^{\prime}$ - E $30^{\circ} 01^{\prime}, 01.11 .2017$, A\&K\&S. 107.

\section{Hygrophoraceae}

43. Cuphophyllus virgineus (Wulfen) Kovalenko: İnönü plateau, in fir and beech 
mixed forest edge, $1250 \mathrm{~m}, \mathrm{~N} 40^{\circ} 34^{\prime}$ - E $29^{\circ}$ $59 ', 10.10 .2016$, A. 6535.

44. Hygrocybe coccinea (Schaeff.) P. Kumm.: İnönü plateau, in fir and beech mixed forest edge, $1120 \mathrm{~m}, \mathrm{~N} 40^{\circ} 33^{\prime}-\mathrm{E} 30^{\circ}$ 00', 29.10.2015, A\&HA. 200.

45. Hygrocybe glutinipes (J.E. Lange) R. Haller Aar.: İnönü plateau, on beech stump, $1120 \mathrm{~m}, \mathrm{~N} 40^{\circ} 34^{\prime}$ - E $29^{\circ}$ 59', 15.09.2016, A. 6466.

46. Hygrocybe punicea (Fr.) P. Kumm.: İnönü plateau, in fir forest edge, $1150 \mathrm{~m}, \mathrm{~N}$ $40^{\circ} 34^{\prime}$ - E $29^{\circ} 59^{\prime}, 27.10 .2015$, A\&HA. 125.

47. Hygrophorus chrysodon (Batsch) Fr.: Ercova plateau, in fir forest, $900 \mathrm{~m}, \mathrm{~N} 40^{\circ}$ 34' - E $29^{\circ}$ 56', 01.10.2015, A.\&HA. 032.

48. Hygrophorus erubescens (Fr.) Fr.: İnönü plateau, in fir and beech mixed forest, $1160 \mathrm{~m}, \mathrm{~N} 40^{\circ} 34^{\prime}-\mathrm{E} 30^{\circ} 00^{\prime}, 30.10 .2015$, A\&HA. 216.

49. Hygrophorus pudorinus (Fr.) Fr.: İnönü plateau, in fir and beech mixed forest edge, $1150 \mathrm{~m}, \mathrm{~N} 40^{\circ} 34^{\prime}-\mathrm{E} 29^{\circ} 59^{\prime}$, 27.10.2015, A\&HA. 122.

\section{Hymenogastraceae}

50. Galerina marginata (Batsch) Kühner: İnönü plateau, on fir stump, $1150 \mathrm{~m}$, $\mathrm{N} 40^{\circ} 33^{\prime}$ - E $30^{\circ} 00^{\prime}, 25.10 .2015$, A\&HA. 084 .

\section{Inocybaceae}

51. Crepidotus epibryus (Fr.) Quél.: İnönü plateau, on beech leaves, $1120 \mathrm{~m}, \mathrm{~N}$ $40^{\circ} 33^{\prime}$ - E $30^{\circ} 00^{\prime}, 29.10 .2015$, A\&HA. 170. 52. Crepidotus variabilis (Pers.) P. Kumm.: İnönü plateau, on beech stump, $1100 \mathrm{~m}, \mathrm{~N} 40^{\circ} 33^{\prime}-\mathrm{E} 30^{\circ} 00^{\prime}, 25.10 .2015$, A\&HA. 086.

53. Inocybe flocculosa Sacc.: Pamukova, in fir forest, $700 \mathrm{~m}, \mathrm{~N} 40^{\circ} 32^{\prime}-\mathrm{E} 30^{\circ} 06^{\prime}$, 25.10.2015, A\&HA. 260.

54. Inocybe geophylla (Bull.) P. Kumm.: Ercova plateau, in fir forest, $1180 \mathrm{~m}, \mathrm{~N} 40^{\circ}$ 34' - E 29 56', 01.10.2015, A\&HA. 040; İnönü plateau, in fir forest, $1150 \mathrm{~m}, \mathrm{~N} 40^{\circ}$ 33' - E 29 59', 01.11.2017, A\&K\&S. 116; Ercova plateau, in fir forest, $1140 \mathrm{~m}, \mathrm{~N}$ $40^{\circ} 34^{\prime}$ - E $30^{\circ} 01^{\prime}, 30.10 .2017$, A\&K\&S. 083.

55. Inocybe nitidiuscula (Britzelm.) Lapl.: İnönü plateau, in fir forest, $1150 \mathrm{~m}, \mathrm{~N}$ $40^{\circ} 34^{\prime}$ - E $29^{\circ} 59^{\prime}, 27.10 .2015$, A\&HA. 121.

\section{Marasmiaceae}

56. Marasmius oreades (Bolton) Fr.: İnönü plateau, in meadow, $1190 \mathrm{~m}, \mathrm{~N} 40^{\circ} 34^{\prime}$ - E $30^{\circ} 00^{\prime}, 30.09 .2015$, A\&HA. 003;

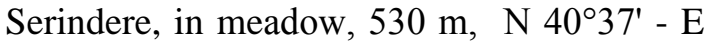
$30^{\circ} 01 ', \quad 01.11 .2017, \mathrm{~A} \& \mathrm{~K} \& S .127$; İnönü plateau, fir and beech mixed forest, $1120 \mathrm{~m}$, $\mathrm{N} 40^{\circ} 33^{\prime}$ - E $30^{\circ} 01 '$, 01.11.2017, A\&K\&S. 109.

57. Marasmius rotula (Scop.) Fr.: İnönü plateau, on fallen beech branch, $1120 \mathrm{~m}, \mathrm{~N}$ $40^{\circ} 33^{\prime}$ - E $30^{\circ} 00^{\prime}, 29.10 .2015$, A\&HA. 167.

\section{Mycenaceae}

58. Mycena crocata (Schrad.) P. Kumm.: İnönü plateau, on beech stump, $1170 \mathrm{~m}, \mathrm{~N}$ $40^{\circ} 34^{\prime}$ - E $30^{\circ} 00^{\prime}, 30.10 .2015$, A\&HA. 216.

59. Mycena epipterygia (Scop.) Gray: Ercova plateau, on fir stump, $1180 \mathrm{~m}, \mathrm{~N} 40^{\circ}$ 34' - E $29^{\circ}$ 56', 01.10.2015, A\&HA. 039; Ercova plateau, on fir stump, $1170 \mathrm{~m}, \mathrm{~N} 40^{\circ}$ $31^{\prime}$ - E 295 56', 29.10.2017, A\&K\&S. 013; Ercova plateau, on fir stump, $1090 \mathrm{~m}, \mathrm{~N} 40^{\circ}$ 34' - E $39^{\circ} 01^{\prime}, 30.10 .2017$, A\&K\&S. 097; İnönü plateau, on fir stump, $1150 \mathrm{~m}, \mathrm{~N} 40^{\circ}$ 33' - E $29^{\circ} 59^{\prime}, 01.11 .2017$, A\&K\&S. 112.

60. Mycena pura (Pers.) P. Kumm.: Ercova plateau, in fir forest, $1150 \mathrm{~m}, \mathrm{~N} 40^{\circ}$ 32' - E $29^{\circ} 55^{\prime}, 01.10 .2015$, A\&HA. 015.

61. Panellus mitis (Pers.) Singer: İnönü plateau, on fir stump, $1140 \mathrm{~m}, \mathrm{~N} 40^{\circ} 34^{\prime}$ - E $29^{\circ} 59^{\prime}, 27.10 .2015$, A\&HA. 119.

\section{Omphalotaceae}

62. Gymnopus androsaceus (L.) DellaMaggiora \& Trassinelli: Ercova plateau, on fallen fir branch, $1175 \mathrm{~m}, \mathrm{~N} 40^{\circ}$ $32^{\prime}$ - E $29^{\circ} 56^{\prime}, 14.09 .2016$, A. 6469.

63. Gymnopus dryophilus (Bull.) Murrill: Pamukova, in fir forest, $700 \mathrm{~m}, \mathrm{~N}$ $40^{\circ} 32^{\prime}$ - E $30^{\circ} 06^{\prime}, 30.10 .2015$, A\&HA. 254.

64. Marasmiellus ramealis (Bull.) Singer: İnönü plateau, on beech stump, 1135 $\mathrm{m}, \mathrm{N} 40^{\circ} 33^{\prime}$ - E $30^{\circ} 00^{\prime}, 10.10 .2016$, A. 6576.

65. Mycetinis alliaceus (Jacq.) Earleex A.W. Wilson \&Desjardin: İnönü plateau, on fallen beech branch, $1130 \mathrm{~m}, \mathrm{~N} 40^{\circ} 33^{\prime}-\mathrm{E}$ $30^{\circ} 00^{\prime}, 10.10 .2016$, A. 6519 .

66. Armillaria mellea (Vahl) P. Kumm.: Kartepe, on apple stump, $120 \mathrm{~m}, \mathrm{~N} 40^{\circ} 41^{\prime}$ E $30^{\circ} 04^{\prime}, 02.10 .2015$, A\&HA. 065.

67. Flammulina velutipes (Curtis) Singer: Kartepe, on apple stump, $120 \mathrm{~m}, \mathrm{~N}$ $40^{\circ} 41^{\prime}$ - E $30^{\circ} 04 ', 02.10 .2015$, A\&HA. 064. 
68. Hymenopellis radicata (Relhan) R.H. Petersen: İnönü plateau, in fir and beech mixed forest, $1120 \mathrm{~m}, \mathrm{~N} 40^{\circ} 34^{\prime}-\mathrm{E} 29^{\circ} 59^{\prime}$, 15.09.2016, A. 6480.

69. Mucidula mucida (Schrad.) Pat.: İnönü plateau, on beech stump, $1100 \mathrm{~m}, \mathrm{~N}$ $40^{\circ} 33^{\prime}$ - E $30^{\circ} 00^{\prime}, 25.10 .2015$, A\&HA. 091.

70. Oudemansiella melanotricha (Dörfelt) M.M. Moser: İnönü plateau, on fir root, $1120 \mathrm{~m}, \mathrm{~N} 40^{\circ} 34^{\prime}$ - E $29^{\circ} 59^{\prime}$, 27.10.2015, A\&HA. 129.

\section{Pleurotaceae}

71. Pleurotus ostreatus (Jacq.) P. Kumm.: Serindere, on beech stump, $900 \mathrm{~m}$, $\mathrm{N} 40^{\circ} 37^{\prime}$ - E $30^{\circ} 00^{\prime}, 27.10 .2015$, A\&HA. 110.

\section{Pluteaceae}

72. Pluteus atromarginatus (Konrad) Kühner: İnönü plateau, on fir stump, 1150 m, N $40^{\circ} 34^{\prime}$ - E $29^{\circ} 59^{\prime}, 27.10 .2015$, A\&HA. 134.

\section{Psathyrellaceae}

73. Coprinellus disseminatus (Pers.) J.E. Lange: Balaban, on plum stump, $200 \mathrm{~m}, \mathrm{~N}$ $40^{\circ} 41^{\prime}$ - E $30^{\circ} 05^{\prime}, 02.10 .2015$, A\&HA. 069.

74. Coprinellus micaceus (Bull.) Vilgalys, Hopple \& Jacq. Johnson: İnönü plateau, on rotten beech stump, $1140 \mathrm{~m}, \mathrm{~N}$ $40^{\circ} 33^{\prime}$ - E $30^{\circ} 00^{\prime}, 10.10 .2016$, A. 6546.

75. Psathyrella candolleana (Fr.) Maire: İnönü plateau, near road, $1080 \mathrm{~m}, \mathrm{~N} \mathrm{40} 34^{\circ}$ E $30^{\circ} 00^{\prime}, 10.10 .2016$, A. 6465.

\section{Rickenellaceae}

76. Rickenella fibula (Bull.) Raithelh.: İnönü plateau, amanog mosses, $1150 \mathrm{~m}, \mathrm{~N}$ $40^{\circ} 34^{\prime}$ - E 295 59', 27.10.2015, A\&HA. 137.

\section{Schizophyllaceae}

77. Schizophyllum commune Fr.: İnönü plateau, on fallen beech branch, $1100 \mathrm{~m}, \mathrm{~N}$ $40^{\circ} 33^{\prime}$ - E $30^{\circ} 00^{\prime}, 02.10 .2015$, A\&HA. 049.

\section{Strophariaceae}

78. Hypholoma fasciculare (Huds.) P. Kumm.: İnönü plateau, on fir stump, 1160 m, N 40 34' - E 29 59', A\&HA. 149.

79. Hypholoma lateritium (Schaeff.) P. Kumm.: İnönü plateau, on beech stump, $1100 \mathrm{~m}, \mathrm{~N} 40^{\circ} 33^{\prime}-\mathrm{E} 30^{\circ} 00^{\prime}, 25.10 .2015$, A\&HA. 087.

80. Pholiota limonella (Peck) Sacc.: Ercova plateau, on fir stump, $1160 \mathrm{~m}, \mathrm{~N} 40^{\circ}$ 32' - E 295 56', 01.10.2015, A\&HA. 017.

81. Stropharia aeruginosa (Curtis) Quél.: İnönü plateau, in fir forest edge, 1150 m, N 40 34' - E 29 59', 28.10.2015, A\&HA. 147.

\section{Tricholomataceae}

82. Clitocybe odora (Bull.) P. Kumm.: Pamukova, in fir forest, $650 \mathrm{~m}, \mathrm{~N} 40^{\circ} 32^{\prime}$ - E $30^{\circ} 06 ', 30.10 .2015$, A\&HA. 253.

83. Clitocybe nebularis (Batsch) $\mathrm{P}$. Kumm.: İnönü plateau, in fir forest, $1130 \mathrm{~m}$, N 40 $33^{\prime}$ - E $29^{\circ} 59^{\prime}, 26.10 .2015$, A\&HA. 106.

84. Lepista nuda (Bull.) Cooke: İnönü plateau, in fir forest edge, $1160 \mathrm{~m}, \mathrm{~N} 40^{\circ} 34^{\prime}$ - E $29^{\circ}$ 59', 27.10.2015, A\&HA. 140; Ercova plateau, in fir forest, $1170 \mathrm{~m}, \mathrm{~N} 40^{\circ} 31^{\prime}$ - E $29^{\circ} 56$ ', 29.10.2017, A\&K\&S. 004; İnönü plateau, in fir forest, $1150 \mathrm{~m}, \mathrm{~N} 40^{\circ} 33^{\prime}-\mathrm{E}$ $29^{\circ} 59^{\prime}, 30.10 .2017$, A\&K\&S. 029.

85. Pseudoclitocybe cyathiformis (Bull.) Singer: İnönü plateau, in fir and beech mixed forest, $1120 \mathrm{~m}, \mathrm{~N} 40^{\circ} 34^{\prime}-\mathrm{E} 29^{\circ} 59^{\prime}$, 15.09.2016, A. 6473.

86. Tricholoma bufonium (Pers.) Gillet: Pamukova, in fir forest, $650 \mathrm{~m}, \mathrm{~N} 40^{\circ} 32^{\prime}$ - E $30^{\circ} 06 ', 30.10 .2015$, A\&HA. 249.

87. Tricholoma sulphureum (Bull.) P. Kumm.: İnönü plateau, in fir forest, $1180 \mathrm{~m}$, $\mathrm{N} 40^{\circ} 33^{\prime}$ - E $30^{\circ} 00^{\prime}, 10.10 .2015$, A. 6550.

88. Tricholoma terreum (Schaeff.) P. Kumm.: Pamukova, in fir forest, $700 \mathrm{~m}, \mathrm{~N}$ $40^{\circ} 32^{\prime}$ - E $30^{\circ} 06^{\prime}, 30.10 .2015$, A\&HA. 244; Ercova plateau, in fir forest, $1085 \mathrm{~m}, \mathrm{~N} 40^{\circ}$ $34^{\prime}$ - E $30^{\circ} 01^{\prime} 30.10 .2017$, A\&K\&S. 072; Ercova plateau, in fir forest, $1102 \mathrm{~m}, \mathrm{~N} 40^{\circ}$ $33^{\prime}$ - E $30^{\circ} 01^{\prime}, 30.10 .2017$, A\&K\&S. 093; İnönü plateau, in beech forest, $1110 \mathrm{~m}, \mathrm{~N} 40^{\circ}$ $33^{\prime}$ - E $30^{\circ} 01^{\prime}, 01.11 .2017$, A\&K\&S. 111.

89. Tricholoma portentosum (Fr.) Quél.: Ercova plateau, in fir forest, $1175 \mathrm{~m}, \mathrm{~N} 40^{\circ}$ $32 '$ - E $29^{\circ} 56^{\prime}, 01.10 .2015$, A\&HA. 029.

90. Tricholoma ustaloides Romagn.: İnönü plateau, in fir and beech mixed forest, $1130 \mathrm{~m}, \mathrm{~N} 40^{\circ} 33^{\prime}-\mathrm{E} 30^{\circ} 00^{\prime}, 29.10 .2015$, A\&HA. 183.

91. Tricholomopsis rutilans (Schaeff.) Singer: Pamukova, on fir stump, $710 \mathrm{~m}, \mathrm{~N}$ $40^{\circ} 32^{\prime}$ - E $30^{\circ} 06^{\prime}, 30.10 .2015$, A\&HA. 241.

\section{Tubariaceae}

92. Tubaria conspersa (Pers.) Fayod: Pamukova, in fir forest, $710 \mathrm{~m}, \mathrm{~N} 40^{\circ} 32^{\prime}-\mathrm{E}$ $30^{\circ} 06^{\prime}, 30.10 .2015$, A.\&HA. 245.

\section{Auriculariales}

Incertae sedis 
93. Pseudohydnum gelatinosum (Scop.) P. Karst.: İnönü plateau, on fir stump, 1140 $\mathrm{m}, \mathrm{N} 40^{\circ} 33^{\prime}$ - E 29० 59', 26.10.2015, A\&HA. 099.

\section{Boletales}

\section{Boletaceae}

94. Boletus erythropus Pers.: İnönü plateau, in fir forest, $1150 \mathrm{~m}, \mathrm{~N} 40^{\circ} 34^{\prime}-\mathrm{E}$ $29^{\circ} 59^{\prime}, 28.10 .2015$, A\&HA. 144.

95. Caloboletus calopus (Pers.) Vizzini: İnönü plateau, in fir and beech mixed forest, $1120 \mathrm{~m}, \mathrm{~N} 40^{\circ} 33^{\prime}$ - E $30^{\circ} 00^{\prime}, 29.10 .2015$, A\&HA. 180.

96. Imleria badia (Fr.) Vizzini: İnönü plateau, in fir and beech mixed forest, 1140 $\mathrm{m}, \mathrm{N} 40^{\circ} 33^{\prime}$ - E $30^{\circ} 00^{\prime}, 10.10 .2016$, A. 6547.

97. Leccinum aurantiacum (Bull.) Gray: İnönü plateau, in fir and beech mixed forest, $1140 \mathrm{~m}, \mathrm{~N} 40^{\circ} 33^{\prime}$ - E $30^{\circ} 00^{\prime}, 10.10 .2016$, A. 6527.

98. Leccinum pseudoscabrum (Kallenb.) Šutara Gray: İnönü plateau, in fir and beech mixed forest, $1120 \mathrm{~m}, \mathrm{~N} 40^{\circ} 34^{\prime}$ - E $29^{\circ} 59^{\prime}$, 15.09.2016, A. 6478.

99. Strobilomyces strobilaceus (Scop.) Berk.: İnönü plateau, in fir and beech mixed forest, $1125 \mathrm{~m}, \mathrm{~N} 40^{\circ} 34^{\prime}$ - E $30^{\circ} 00^{\prime}$, 30.10.2015, A\&HA. 218.

\section{Paxillaceae}

100. Paxillus involutus (Batsch) Fr.: Pamukova, in fir forest edge, $690 \mathrm{~m}, \mathrm{~N} 40^{\circ}$ $32^{\prime}$ - E $30^{\circ} 06 ', 30.10 .2015$, A\&HA. 240.

\section{Sclerodermataceae}

101. Scleroderma bovista Fr.: Yuvacık Dam, near road, $190 \mathrm{~m}, \mathrm{~N} 40^{\circ} 38^{\prime}$ - E $29^{\circ} 56^{\prime}$, 02.10.2015, A\&HA. 054.

102. Scleroderma citrinum Pers.: Karaaslan recreational area, near road, 300 $\mathrm{m}, \mathrm{N} 40^{\circ} 38^{\prime}$ - E 29० 56', 02.10.2015, A\&HA. 045.

\section{Suillaceae}

103. Suillus bovinus (L.) Roussel: İnönü plateau, under pine, $1080 \mathrm{~m}, \mathrm{~N} 40^{\circ} 34^{\prime}-\mathrm{E}$ $30^{\circ} 00^{\prime}, 10.10 .2016$, A. 6552.

104. Suillus luteus (L.) Roussel: İnönü plateau, under pine, $1070 \mathrm{~m}, \mathrm{~N} 40^{\circ} 34^{\prime}-\mathrm{E}$ $30^{\circ} 00^{\prime}, 15.09 .2016$, A. 6462.

\section{Cantharellales}

\section{Cantharellaceae}

105. Cantharellus cibarius Fr.: İnönü plateau, fir and beech mixed forest, $1120 \mathrm{~m}$, $\mathrm{N} 40^{\circ} 33^{\prime}$ - E $30^{\circ} 00^{\prime}$, 29.10.2015, A\&HA.
209; Ercova plateau, in fir forest, $1085 \mathrm{~m}, \mathrm{~N}$ $40^{\circ} 34^{\prime}-$ E $30^{\circ} 01^{\prime}, 30.10 .2017$, A\&K\&S. 063; Ercova plateau, in fir forest, $1102 \mathrm{~m}, \mathrm{~N}$ $40^{\circ} 33^{\prime}$ - E $30^{\circ} 01^{\prime}$ E 30.10 .2017 , A\&K\&S. 090.

106. Craterellus cornucopioides (L.) Pers.: İnönü plateau, fir and beech mixed forest, $1170 \mathrm{~m}, \mathrm{~N} 40^{\circ} 34^{\prime}-\mathrm{E} 30^{\circ} 00^{\prime}$, 30.10.2015, A\&HA. 230; İnönü plateau, fir and beech mixed forest, $1120 \mathrm{~m}, \mathrm{~N} 40^{\circ} 33^{\prime}$ E $30^{\circ} 01^{\prime}, 01.11 .2017$, A\&K\&S. 114.

107. Craterellus tubaeformis (Fr.) Quél.: İnönü plateau, fir and beech mixed forest edge, $1120 \mathrm{~m}, \mathrm{~N} 40^{\circ} 34^{\prime}-\mathrm{E} 29^{\circ} 5^{\prime}$, 15.09.2016, A. 6463.

108. Pseudocraterellus undulatus (Pers.) Rauschert: İnönü plateau, fir and beech mixed forest, $1120 \mathrm{~m}, \mathrm{~N} 40^{\circ} 33^{\prime}$ - E $30^{\circ} 00^{\prime}$, 10.10.2016, A. 6555.

\section{Clavulinaceae}

109. Clavulina cinerea (Bull.) J. Schröt.: İnönü plateau, fir forest, $1150 \mathrm{~m}, \mathrm{~N} 40^{\circ} 34^{\prime}$ E $29^{\circ} 59^{\prime}, 27.10 .2015$, A\&HA. 120.

110. Clavulina coralloides (L.) J. Schröt.: İnönü plateau, fir forest, $1160 \mathrm{~m}, \mathrm{~N} 40^{\circ} 34^{\prime}$ E $29^{\circ} 59^{\prime}, 28.10 .2015$, A\&HA. 142.

\section{Hydnaceae}

111. Hydnum repandum L.: İnönü plateau, in beech forest, $1100 \mathrm{~m}, \mathrm{~N} 40^{\circ} 33^{\prime}-\mathrm{E} 30^{\circ}$ 00', 25.10.2015, A\&HA. 090.

\section{Dacrymycetales \\ Dacrymycetaceae}

112. Calocera cornea (Batsch) Fr.: İnönü plateau, on beech stump, $1120 \mathrm{~m}, \mathrm{~N} 40^{\circ} 33^{\prime}$ E $30^{\circ} 00^{\prime}, 29.10 .2015$, A\&HA. 177.

113. Calocera viscosa (Pers.) Fr.: Ercova plateau, on fir stump, $1160 \mathrm{~m}, \mathrm{~N} 40^{\circ} 32^{\prime}-\mathrm{E}$ $29^{\circ} 55^{\prime}, 01.10 .2015$, A\&HA. 020.

\section{Gomphales}

\section{Clavariadelphaceae}

114. Clavariadelphus truncatus Donk: Ercova plateau, in fir forest, $1160 \mathrm{~m}, \mathrm{~N} 40^{\circ}$ $32^{\prime}$ - E $29^{\circ} 55^{\prime}, 01.10 .2015$, A\&HA. 019.

\section{Hymenochaetales \\ Incertae sedis}

115. Trichaptum biforme (Fr.) Ryvarden: İnönü plateau, on beech stump, $1130 \mathrm{~m}, \mathrm{~N}$ $40^{\circ} 33^{\prime}$ - E $30^{\circ} 00^{\prime}, 29.10 .2015$, A\&HA. 173; Kartepe, on beech stump, 254m, N 40 $41^{\circ}$ - E $30^{\circ} 00^{\prime}, 30.10 .2017$, A\&K\&S. 075; İnönü plateau, on beech stump, $1110 \mathrm{~m}, \mathrm{~N} 40^{\circ} 33^{\prime}$ E $30^{\circ} 01 ', 01.11 .2017$, A\&K\&S. 108 .

\section{Phallales}




\section{Phallaceae}

116. Clathrus ruber P. Micheli ex Pers.: Karaaslan recreational area, near road, 300 $\mathrm{m}, \mathrm{N} 40^{\circ} 38^{\prime}$ - E 29० 56', 02.10.2015, A\&HA. 040.

117. Mutinus caninus (Huds.) Fr.: İnönü plateau, in fir and beech mixed forest, 1120 m, N 40 33' - E $30^{\circ} 00^{\prime}, 29.10 .2015$, A\&HA. 177.

118. Phallus impudicus L.: İnönü plateau, in fir ans beech mixed forest, $1150 \mathrm{~m}, \mathrm{~N} 40^{\circ}$ 34' - E $29^{\circ}$ 59', 10.10.2016, A. 6529.

\section{Polyporales}

\section{Fomitopsidaceae}

119. Fomitopsis pinicola (Sw.) P. Karst.: Ercova plateau, on fir, $1160 \mathrm{~m}, \mathrm{~N} 40^{\circ} 32^{\prime}-\mathrm{E}$ $29^{\circ}$ 55', 01.10.2015, A\&HA. 014; Ercova plateau, on fir trunk, $1170 \mathrm{~m}, \mathrm{~N} 40^{\circ} 31^{\prime}-\mathrm{E}$ 29 56', 29.10.2017, A\&K\&S. 007; Ercova

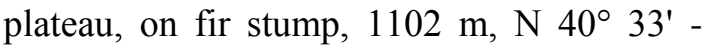
$30^{\circ}$ 01', 30.10.2017, A\&K\&S. 091; İnönü

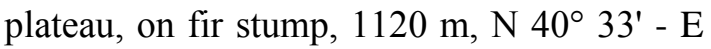
$30^{\circ} 01^{\prime}, 01.11 .2017$, A\&K\&S. 122.

\section{Meruliaceae}

120. Bjerkandera adusta (Willd.) P. Karst.: İnönü plateau, on beech stump, 1175 $\mathrm{m}, \mathrm{N} 40^{\circ} 34^{\prime}$ - E $30^{\circ} 00^{\prime}, 15.04 .2016$, A. 6419; İnönü plateau, on beech stump, 1130 $\mathrm{m}, \mathrm{N} 40^{\circ} 33^{\prime}$ - E $30^{\circ} 00^{\prime}, 10.10 .2016$, A. 6548; Kartepe, on beech stump, 254m, N $40^{\circ}$ 41' - E 30 00', 30.10.2017, A\&K\&S. 074; İnönü plateau, on beech stump, $1110 \mathrm{~m}, \mathrm{~N}$ $40^{\circ} 33^{\prime}$ - E $30^{\circ} 01^{\prime}, 01.11 .2017$, A\&K\&S. 103.

\section{Polyporaceae}

121. Daedaleopsis confragosa (Bolton) J. Schröt.: İnönü plateau, on beech stump, 1130 $\mathrm{m}, \mathrm{N} 40^{\circ} 33^{\prime}$ - E $30^{\circ} 00^{\prime}, 29.10 .2015$, A\&HA. 172.

122. Fomes fomentarius (L.) Fr.: İnönü plateau, on beech stump, $1100 \mathrm{~m}, \mathrm{~N} 40^{\circ} 33^{\prime}$ E $30^{\circ} 00^{\prime}, 25.10 .2015$, A\&HA. 073.

123. Lentinus brumalis (Pers.) Zmitr.: İnönü plateau, on beech stump, $1135 \mathrm{~m}, \mathrm{~N}$ $40^{\circ} 33^{\prime}$ - E $30^{\circ} 00^{\prime}, 10.10 .2016$, A. 6524.

124. Lenzites betulina (L.) Fr.: İnönü plateau, on beech stump, $1120 \mathrm{~m}, \mathrm{~N} 40^{\circ} 33^{\prime}$ E $30^{\circ} 00^{\prime}, 29.10 .2015$, A\&HA. 202.

125. Polyporus varius (Pers.) Fr.: İnönü plateau, on beech stump, $1170 \mathrm{~m}, \mathrm{~N} 40^{\circ} 34^{\prime}$ E $30^{\circ} 00^{\prime}, 30.10 .2015$, A\&HA. 224.
126. Trametes hirsuta (Wulfen) Lloyd: İnönü plateau, on beech stump, $1125 \mathrm{~m}, \mathrm{~N}$ $40^{\circ} 34^{\prime}$ - E 29 59', 15.09.2016, A. 6479;

127. Trametes versicolor (L.) Lloyd: İnönü plateau, on beech stump, $1100 \mathrm{~m}, \mathrm{~N}$ $40^{\circ} 33^{\prime}$ - E $30^{\circ} 00^{\prime}, 25.10 .2015$, A\&HA. 078; İnönü plateau, on beech stump, $1110 \mathrm{~m}, \mathrm{~N}$ $40^{\circ} 33^{\prime}$ - E $30^{\circ} 01^{\prime}, 01.11 .2017$, A\&K\&S. 106.

\section{Sparassidaceae}

128. Sparassis crispa (Wulfen) Fr.: İnönü plateau, on fir root, $1150 \mathrm{~m}, \mathrm{~N} 40^{\circ} 34^{\prime}-\mathrm{E}$ 09 59', 27.10.2015, A\&HA. 124.

\section{Russulales}

\section{Hericiaceae}

129. Hericium cirrhatum (Pers.) Nikol.: İnönü plateau, on beech stump, $1135 \mathrm{~m}, \mathrm{~N}$ $40^{\circ} 33^{\prime}$ - E $30^{\circ} 00^{\prime}, 10.10 .2016$, A. 6521.

130. Hericium coralloides (Scop.) Pers.: İnönü plateau, on fir stump, $1150 \mathrm{~m}, \mathrm{~N} \mathrm{40^{ \circ }}$ 33' - E 29 59', 26.10.2015, A\&HA. 097.

131. Hericium erinaceus (Bull.) Pers.: İnönü plateau, on beech stump, $1180 \mathrm{~m}, \mathrm{~N}$ $40^{\circ} 34^{\prime}$ - E $30^{\circ} 00^{\prime}, 30.10 .2015$, A\&HA. 239.

\section{Incertae sedis}

132. Laeticutis cristata (Schaeff.) Audet: İnönü plateau, in fir forest, $1110 \mathrm{~m}, \mathrm{~N} \mathrm{40^{ \circ }}$ $33^{\prime}$ - E $29^{\circ} 59^{\prime}, 10.10 .2015$, A. 6556.

\section{Russulaceae}

133. Lactarius aurantiacus (Pers.) Gray: İnönü plateau, in fir forest, $1140 \mathrm{~m}, \mathrm{~N} 40^{\circ}$ 33' - E $29^{\circ} 59^{\prime}, 26.10 .2015$, A\&HA. 101.

134. Lactarius blennius (Fr.) Fr.: İnönü plateau, in fir and beech mixed forest, 1170 $\mathrm{m}, \mathrm{N} 40^{\circ} 34^{\prime}$ - E $30^{\circ} 00^{\prime}, 30.10 .2015$, A\&HA. 229.

135. Lactarius deliciosus (L.) Gray: Yuvacık Dam, under pine, $200 \mathrm{~m}, \mathrm{~N} 40^{\circ} 38^{\prime}$ E 29 56', 11.10.2016, A. 6571.

136. Lactarius salmonicolor R. Heim \& Leclair: Ercova plateau, in fir forest, $1175 \mathrm{~m}$, N 40 32' - E 29 56', 01.10.2015, A\&HA. 027; Ercova plateau, in fir forest, $1170 \mathrm{~m}, \mathrm{~N}$ $40^{\circ} 31^{\prime}$ - E 29 56', 29.10.2017, A\&K\&S. 014; Ercova plateau, in fir forest, $1100 \mathrm{~m}, \mathrm{~N}$ $40^{\circ} 33^{\prime}$ - E $30^{\circ} 01^{\prime}$, 30.10.2017; A\&K\&S. 019; Ercova plateau, in fir forest, $1140 \mathrm{~m}, \mathrm{~N}$ $40^{\circ} 34^{\prime}$ - E $30^{\circ} 01^{\prime}, 30.10 .2017$, A\&K\&S. 078; İnönü plateau, in fir forest, $1110 \mathrm{~m}, \mathrm{~N}$ $40^{\circ} 33^{\prime}$ - E $30^{\circ} 01^{\prime}, 01.11 .2017$, A\&K\&S. 101 . 
137. Lactarius vellereus (Fr.) Fr.: İnönü plateau, fir and beech mixed forest, $1120 \mathrm{~m}$, N 40 34' - E 29 59', 15.09.2016, A. 6483.

138. Russula delica Fr.: İnönü plateau, in fir and beech mixed forest edge, $1210 \mathrm{~m}, \mathrm{~N}$ $40^{\circ} 34^{\prime}$ - E $29^{\circ}$ 59', 14.09.2016, A. 6468.

\section{Stereaceae}

139. Stereum hirsutum (Willd.) Pers.: İnönü plateau, on beech stump, $1170 \mathrm{~m}, \mathrm{~N}$ $40^{\circ} 34^{\prime}$ - E $30^{\circ} 00^{\prime}, 30.10 .2015$, A\&HA. 228; 15.04.2016, A. 6413; İnönü plateau, on beech stump, $1110 \mathrm{~m}, \mathrm{~N} 40^{\circ} 33^{\prime}$ - E 3001', 01.11.2017, A\&K\&S. 100; Serindere, on apple stump, $480 \mathrm{~m}, \mathrm{~N} 40^{\circ} 38^{\prime}-\mathrm{E} 30^{\circ} 01^{\prime}$, 01.11.2017, A\&K\&S. 124.

\section{Tremellales}

\section{Tremellaceae}

140. Tremella mesenterica Retz.: İnönü plateau, on beech stump, $1125 \mathrm{~m}, \mathrm{~N} 40^{\circ} 34^{\prime}$ E 29 59', 15.09.2016, A. 6477.

\section{Discussion}

One hundred and forty macrofungi species belonging to ninety genera and fortyfour families within the fourteen order were determined. Thirteen of them belonged to Ascomycota and one hundred and twentyseven to Basidiomycota. All species except Cortinarius caperatus were new for the research area. The distribution of the species in their orders were as follows; Agaricales
79, Russulales and Boletales 11, Polyporales 10, Cantharellales 7, Pezizales 5, Helotiales and Xylariales 4, Phallales 3, Dacrymycetales 2, Auriculariales, Gomphales, Hymenochaetales and Tremellales 1.

In the study area, eighty-nine macrofungi species were terricolous and fifty-one were lignicolous. The most common terricolous macrofungi were Amanita muscaria, Coprinus comatus, Craterellus cornucopioides, Inocybe geophylla, Laccaria laccata, Lactarius aurantiacus, L. salmonicolor, Lepista nuda, Macrolepiota procera, Mycena epipterygia and Tricholoma terreum. Moreover, eight lignicolus macrofungi species (Bisporella citrina, Bjerkandera adusta, Fomes fomentarius, Fomitopsis pinicola, Stereum hirsutum, Trametes versicolor, Trichaptum biforme and Xylaria hypoxylon) were widespread in the research area.

Fifty-four of one hundered and fourty species determined in the study area were edible (Table 1). However, nine species (Cantharellus cibarius, Craterellus cornucopioides, Lactarius deliciosus, L. salmonicolor, Lepista nuda, Macrolepiota procera, Marasmius oreades, Russula delica and Tricholoma terreum) were known and commonly consumed by local people.

Table 1. Edible macrofungi species determined in the research area

\begin{tabular}{lll}
\hline Helvella crispa & Laccaria amethystina & Suillus bovinus \\
\hline Helvella elastica & Laccaria laccata & Cantharellus cibarius \\
\hline Aleuria aurantia & Hygrophorus chrysodon & Craterellus cornucopioides \\
\hline Coprinus comatus & Hygrophorus erubescens & Craterellus tubaeformis \\
\hline Macrolepiota konradii & Hygrophorus pudorinus & Pseudocraterellus undulatus \\
\hline Macrolepiota mastoidea & Marasmius oreades & Clavulina cinerea \\
\hline Macrolepiota procera & Gymnopus dryophilus & Clavulina coralloides \\
\hline Leucoagaricus leucothites & Armillaria mellea & Hydnum repandum \\
\hline Lycoperdon echinatum & Flammulina velutipes & Clavariadelphus truncatus \\
\hline Lycoperdon excipuliforme & Mucidula mucida & Sparassis crispa \\
\hline Lycoperdon molle & Pleurotus ostreatus & Hericium cirrhatum \\
\hline Lycoperdon perlatum & Lepista nuda & Hericium coralloides \\
\hline Lycoperdon pyriforme & Tricholoma terreum & Hericium erinaceus \\
\hline
\end{tabular}


Table 1 (continued)

\begin{tabular}{lll}
\hline Lycoperdon utriforme & Tricholoma portentosum & Laeticutis cristata \\
\hline Amanita rubescens & Tricholomopsis rutilans & Lactarius deliciosus \\
\hline Amanita vaginata & Imleria badia & Lactarius salmonicolor \\
\hline Cortinarius caperatus & Leccinum aurantiacum & Lactarius vellereus \\
\hline Fistulina hepatica & Suillus luteus & Russula delica \\
\hline
\end{tabular}

Fifteen poisonous species (Amanita muscaria, A. pantherina, A. phalloides, Boletus erythropus, Galerina marginata, Hypholoma fasciculare, $H$. lateritium, Inocybe geophylla, Lepiota castanea, $L$. cristata, L. clypeolaria, Mycena pura, Paxillus involutus, Tricholoma bufonium and T. sulphureum) were also determined in the region. Among them, A. phalloides, $G$. marginata and $L$. castanea were deadly poisonous species which contains alpha amanitin (Akata et al., 2015b). No poisoning event has hitherto been reported in the study area as the native people collect and consume only well known edible mushrooms.

The results of the current study were compared with the findings of the investigations compiled in close environs. Number of the determined species and similarity percentages were presented in Table 2. These similarities may be due to the similarities in climate and vegetation.

Table 2. Similarity percentages of the neighboring areas with the research area

\begin{tabular}{lccc}
\hline Investigated areas & Number of species & Common species & Similarity (\%) \\
\hline Bursa (Karamanoğlu and Öder, 1973) & 13 & 5 & 38.46 \\
\hline İnegöl/Bursa (Öztürk et al., 1990) & 22 & 8 & 36.36 \\
\hline Bursa (Solak and Gücin, 1992) & 80 & 26 & 32.50 \\
\hline Trakya (Stojchev et al., 1998) & 67 & 27 & 40.29 \\
\hline Belgrad Forest/İstanbul (Akata, 2017) & 279 & 96 & 34.40 \\
\hline Yalova (Allı et al., 2017) & 92 & 36 & 39.13 \\
\hline
\end{tabular}

\section{Acknowledgements}

The first author is thankful to Ankara University's Central Research Funding Unit (Project no: 16H0430013) for its financial support.

\section{References}

Akata, I. (2017) Macrofungal Diversity of Belgrad Forest (İstanbul). Kastamonu Üniversitesi Orman Fakültesi Dergisi, 17(1), 150-164.

Akata, I., Uzun, Y. (2017) Macrofungi determined in Uzungöl Nature Park (Trabzon). Trakya University Journal of Natural Sciences, 18(1),15-24.

Akata, I., Kabaktepe, Ş., Akgül, H. (2015a) Cortinarius caperatus (Pers.) Fr.,
A New Record For Turkish Mycobiota. Kastamonu Üniversitesi Orman Fakültesi Dergisi, 15(1), 86-89.

Akata, I., Kaya, E., Yılmaz, İ., Bakırcı, S., Bayram, R. (2015b) Türkiye'de Yetişen Alfa Amanitin İçeren Mantarlar, Düzce Tip Fakültesi Dergisi, 17(1), 39-44.

Alkan, S., Kaşı, G., Öztürk, C., Aktaş, S. (2016) Çorum İli'nin Yenir Özellikteki Makromantarları. Türk Tarım Gıda Bilim ve Teknoloji Dergisi, 4(3), 131-138.

Allı, H., Candar, S.S., Akata, I. (2017) Macrofungal Diversity of Yalova Province. Mantar Dergisi, 8(2), 76-84.

Altuntaş, D., Allı, H., Akata, I. (2017) Macrofungi of Kazdağ 1 National Park (Turkey) and its close environs. 
Biological Diversity and Conservation, 10(2), 17-25.

Beşkardeş, V. (2012) Lepidoptera fauna of Yuvacik dam watershed in Kocaeli, Turkey. African Journal of Agricultural Research, 7(11), 1749-1754.

Breitenbach, J., Kränzlin, F. (1986) Fungi of Switzerland, Volume 2, Nongilled Fungi, Verlag Mykologia CH-6000 Luzern 9, Switzerland.

Breitenbach, J., Kränzlin, F. (1991) Fungi of Switzerland, Volume 3, Boletes and Agarics 1. Part, Verlag Mykologia $\mathrm{CH}-$ 6000 Luzern 9, Switzerland.

Breitenbach, J., Kränzlin, F. (1995) Fungi of Switzerland, Volume 4, Agarics 2. Part, Verlag Mykologia CH-6000 Luzern 9, Switzerland.

Breitenbach, J., Kränzlin, F. (2000) Fungi of Switzerland, Volume 5, Agarics 3. Part, Verlag Mykologia CH-6000 Luzern 9, Switzerland.

Calonge, F.D. (1998) Gasteromycetes, I. Lycoperdales, Nidulariales, Phallales, Sclerodermatales, Tulostomatales, Volume 3, Real Jardin Botanico, Stuttgart.

Cannon, P.F., Kirk, P.F. (2008) Fungal families of the World CAB International, Wallingford.

Çobanoğlu, G., Açıkgöz, B., Baloniu, L. (2013) Contributions to lichen diversity of Turkey from the Sarısu area (Kocaeli). Turkish Journal of Botany, 37, 964-969.

Davis, P.H. (1965) Flora of Turkey and the east Aegean islands, Volume 1, Edinburgh Univ Press, Edinburgh.

Demirel, K., Acar, İ., Ömeroğlu Boztepe, G. (2016) Lice (Diyarbakır) Yöresi Makrofunguslar1. Mantar Dergisi 7(1), 29-39.

Doğan, H.H., Kurt, F. (2016) New macrofungi records from Turkey and macrofungal diversity of Pozant1-Adana. Turkish Journal of Botany, 40, 209-217.

Efe A., Aksoy, N., Güneş Özkan, N., Demir Oral, D., Aslan, S. (2013) Yuvacık Baraj1 Havzası'nın (Kocaeli-Sakarya) Florası. Ormancllık Dergisi, 9(2), 56-92.

Hansen, L., Knudsen, H. (1992) Nordic Macromycetes, Volume 2, Polyporales, Boletales, Agaricales, Russulales. Nordsvamp, Copenhagen, Denmark.
Hansen, L., Knudsen, H. (1997) Nordic Macromycetes, Volume 3, Heterobasidoid, Aphyllophoroid, and Gastromycetoid Basidiomycetes, Nordsvamp, Copenhagen, Denmark.

Hansen, L., Knudsen, H. (2000) Nordic Macromycetes, Volume 1, Ascomycetes, Nordsvamp, Copenhagen, Denmark.

Heilmann-Clausen, J., Verbeken, A., Vesterholt, J. (1998) The Genus Lactarius (Fungi of Northern Europe), Volume 2, Danish Mycological Society, Copenhagen, Denmark.

Karamanoğlu, K., Öder, N. (1973) Bursa ili ve çevresinde yetişen bazı şapkalı mantarlar. Ankara Üniversitesi Eczacıllk Fakültesi Mecmuası, 3(13), 13-33.

Keten, A., Beşkardeş, V., Arslangündogdu Z. (2010) Observation on ornithofauna of Kocaeli-Yuvacik dam watershed in Turkey. Journal of Environmental Biology, 31, 189-195.

Kirk, P.F., Cannon, P.F., Minter, D.W., Stalpers, J.A. (2008) Dictionary of the fungi 10th ed. CAB International, Wallingford, UK.

Kränzlin, F. (2005) Fungi of Switzerland, Volume 6, Russulaceae, 2. Verlag Mykologia, Switzerland.

Öztürk, A., Demirel, K., Arık, İ.H. (1990) İnegöl (Bursa) Çevresinde Yetişen Zehirli ve Yenen Mantarlar Üzerinde Sistematik, Morfolojik ve Ekolojik İncelemeler. Yüzüncü Yıl Üniversitesi, Fen Bilimleri Dergisi, 1(1), 27-38.

Öztürk, C., Pamukçu, D., Aktaş, S. (2017) Macrofungi of Nallihan (Ankara) District. Mantar Dergisi, 8(1), 60- 67.

Pegler, D.N., Roberts, P.J., Spooner, B.M. (1997) British Chanterelles and Tooth Fungi, Royal Botanic Gardens, Kiew.

Ryvarden, L., Gilbertson, R.L. (1993) European Polypores Vol: 1-2, Synopsis Fungorum 6, Fungiflora, Oslo, Norway.

Sesli, E., Denchev, C.M. (2008) Checklists of the myxomycetes, larger ascomycetes, and larger basidiomycetes in Turkey. Mycotaxon, 106, 65-67.

Sesli, E., Türkekul, İ., Akata, I., Niskanen, T. (2016) New records of Basidiomycota from Trabzon, Tokat. and İstanbul provinces in Turkey, Turkish Journal of Botany 40, 531-545. 
Solak, M.H., Gücin, F. (1992) Bursa

Yöresinden Türkiye için Yeni

Makrofungus Türleri ve Yörede

Belirlenen Diğer Makrofunguslar. Turkish Journal of Botany, 16, 335-346.

Stojchev, G., Asan, A., Gücin, F. (1998)

Some macrofungi species of European part of Turkey, Turkish Journal of Botany, 24, 341-348. 